\title{
Near-Field Antennas Integrated With Scanning Probes for THz to Visible Microscopy: Scale Modeling and Limitations on Performance
}

\author{
Björn Rosner, Student Member, IEEE, John Peck, Student Member, IEEE, and Daniel van der Weide, Member, IEEE
}

\begin{abstract}
A promising approach to localized spectroscopy at far-infrared $(\lambda>5 \mu \mathrm{m})$ and terahertz frequencies $(100-$ $3000 \mathrm{GHz}$ ) is with scanning probe microscopes by transducing far-field radiation to the near field with a field confinement structure-a near-field antenna. Here we discuss the background of this idea and show encouraging results from a scale-model experiment using 3-GHz microwave radiation. We also present experiments to scale antennas to visible wavelengths and we discuss limits to scale modeling.
\end{abstract}

Index Terms-Atomic force microscopy, coaxial aperture antennas, infrared spectroscopy, millimeter-wave antennas.

\section{INTRODUCTION}

$\mathbf{T}$ HE SUBVISIBLE electromagnetic spectrum has not enjoyed the wide range of results obtained in visible microscopy because the diffraction limit for focusing wavelengths from 1.6 to over $300 \mu \mathrm{m}$ precludes achieving microscopically interesting resolution with ordinary optics. Although recent developments have stimulated great interest [1]-[4], the wealth of spectroscopic information available in this regime has thus remained untapped except in collective (far-field) measurements, such as Fourier-transform infrared spectroscopy (FTIR), which is a mainstay of chemical analysis.

To achieve a better spatial resolution than possible with a far-field microscope, the concept of near-field microscopy was introduced in 1928 [5]. Antenna radiation emerges from the static, reactive near-field region via the radiating near-field region to the far field. The static reactive near-field region can be much smaller than the corresponding wavelength and can be used to locally illuminate a sample or device under test (DUT). The DUT needs to be very close to the tip $(\ll \lambda)$ so that the reactive near field overlaps with its boundary and is raster scanned with respect to the near-field source, resulting in better spatial resolution than possible with a far-field microscope. Ash and Nicholls demonstrated this technique in 1972 using $\lambda=3 \mathrm{~cm}$ microwaves confined to a subwavelength aperture, achieving

\footnotetext{
Manuscript received August 3, 2001; revised October 29, 2001. This work was supported in part by the Cornell Nanofabrication Facility (a member of the National Nanofabrication Users Network) which is supported by the National Science Foundation under Grant ECS-9731293, its users, Cornell University, and Industrial Affiliates. The work was also supported by DARPA (Ultrafast Electronics and Hybrid Computing), ONR (MURI), and NSF (PECASE and SGER).

The authors are with the Department of Electrical and Computer Engineering, University of Wisconsin-Madison, Madison WI 53706 USA (e-mail: danvdw@engr.wisc.edu).

Publisher Item Identifier S 0018-926X(02)05440-6.
}

$\lambda / 60$ resolution [6]. Following this demonstration, researchers developed many of the principles of near-field microwave and far infrared (FIR) scanning using both aperture and antenna probes for millimeter-scale samples [7]-[10]. Lately, the advent of scanning probe microscopy (SPM) [11], [12] has opened this field to optical wavelengths, since the distance between sample and near-field source can be more tightly controlled with force or current feedback. SPM is now also employed in microwave near-field microscopy mode for its superior distance control and ability to map topography simultaneously [13]. This multifunctional ability is particularly important in submicrometer field resolution, since the tip-sample distance should be stabilized independent of the field measurement.

Near-field imaging has been most prominent in the visible, where near-field scanning optical microscopy (NSOM) [14]-[19] confines light within a subwavelength aperture (or scatters it with a subwavelength tip [20]-[22]) to excite or detect evanescent waves on a sample. However, there are myriad electromagnetic phenomena with characteristic frequencies below the visible but above the radio, including integrated circuit (IC) electromagnetic fields (between $100 \mathrm{MHz}$ and $300 \mathrm{GHz}$ ), biological membrane absorption in the FIR range of $100 \mathrm{GHz}$ to $30 \mathrm{THz}(10 \mu \mathrm{m})$, molecular rotational or vibrational absorption (also primarily in FIR), and conductive or dielectric properties of materials. Just as far-field optics enable traditional spectroscopy, which measures the collective response of large numbers of elements (such as molecular electric dipoles), near-field techniques now enable localized spectroscopy.

In the FIR and IR bands, a wide range of techniques is employed, with most ideas being ported from the visible or the RF regime due to a lack of unique and optimal solutions for the infrared. Modern aperture probes for the microwave through FIR regime include both conventional open-ended hollow waveguides [23], [24] and specially fabricated tapered focusing cones [1]. Aperture probes are the predominant near-field source in the visible and have the advantage of being better shielded than point scattering antennas. Since their resolution and throughput depend inversely on wavelength, however, they are not suited for achieving high spatial resolution in the microwave regime. The opposite of an aperture probe is an apertureless one: a sharp needle probe can serve to scatter the near-field energy in a nanometer-scale region of an illuminated surface. A few groups [25], [26] have adapted this technique to probe samples at the $10.6-\mu \mathrm{m}\left(\mathrm{CO}_{2}\right.$ laser) wavelength since it readily accommodates conventional SPM tips, which serve double duty as scattering probes and topography sensors. Other 


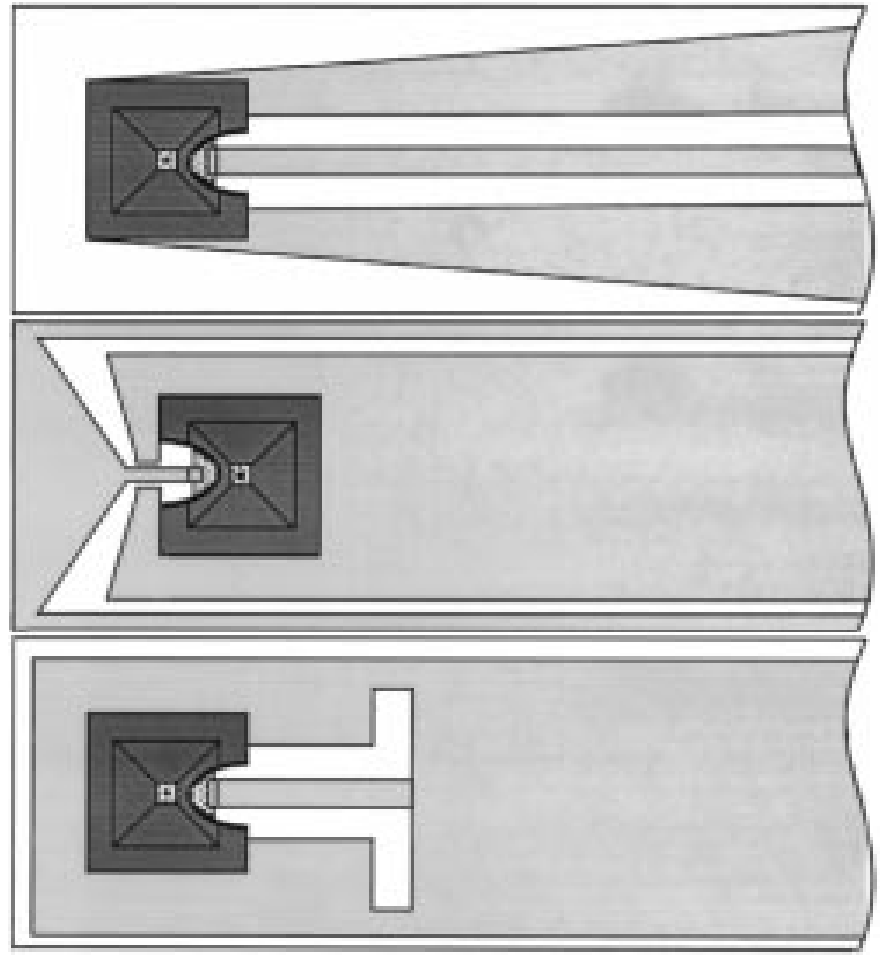

Fig. 1. SPM cantilevers incorporating waveguides and antennas feeding near-zone coaxial tips. From top to bottom: coplanar waveguide, slot-V antenna, and resonant slot antenna.

groups have used total internal reflection tips [27] or heated tips as black body sources for local FTIR spectra [4].

The most richly developed approaches to subvisible microscopy, however, use structured near-field waveguides and antennas. Various configurations that incorporate waveguides and antennas on cantilevers can be envisioned (Fig. 1) [28]. Seminal work demonstrating subwavelength field resolution with a coaxial tip was published by Fee et al. in 1989 [29] at about the same time as Keilmann independently submitted a U.S. patent application on localizing optical radiation with a tapered coaxial tip [30]. The advantage of using a coaxial waveguide for near-field imaging in the subvisible regime is that the shield greatly reduces interference from stray radiation while enabling a noncutoff transverse electromagnetic (TEM) mode of wave propagation up to or away from the tip. If the tip is sufficiently sharp, it can also function independently as an SPM topography probe, either in force [13], [31] or current feedback [32].

\section{BOWTIE ANTENNAS FOR NEAR-FIELD IR AND FIR MICROSCOPY}

While these coaxial waveguides are very efficient in the microwave regime, new techniques need to be employed at higher frequencies where TEM waveguides are not available. The potential of antennas as far field to near-field transducers for nearfield microscopy has recently been pointed out in scale model studies by Grober et al. [33], [34]. Unlike in conventional antenna applications, where they serve together with a feed line as either source or detector for radiation, they are used here without feeds. The antenna acts as a transducer that converts incident freely propagating radiation to current and reradiates this energy again, originating from a near field spot, so it can have a conversion efficiency far exceeding that of cut-off aperture probes.

We choose a three-dimensional (3-D) bowtie antenna since it is easy to pattern, consisting only of two triangular metal patches that can be lithographically defined with high resolution, even for relatively short wavelengths. Its broad-band character simplifies design and allows application over a wide wavelength range. Furthermore, it is particularly well suited for microfabrication on pyramidal SPM tips, while the highest field concentration lies at the center of the antenna, coincident with the sharp SPM tip.

Here we examine a bowtie patterned on a silicon-oxy nitride tip and cantilever. These $\mathrm{SiO}_{x} \mathrm{~N}_{y}$ tips have a pyramidal shape since they are formed in a silicon mold produced by anisotropic anisotropic potassium hydroxide $(\mathrm{KOH})$ etching. This tip geometry is often applied in SPM and it lends itself to maskless antenna fabrication as shown in the fabrication suggestion in Fig. 2. Here, it can be seen how a bowtie shaped metallization with nanometer gaps between the flares can be realized without using electron beam lithography. By positioning the tip at an angle to the stream of metal in an evaporator, it is possible to coat only one side of the pyramid. The entire tip is coated from the tip side with a thin layer of cantilever material $\left(\mathrm{SiO}_{x} \mathrm{~N}_{y}\right)$ by plasma-enhanced chemical vapor deposition (PECVD). This layer determines the spacing between the two patches of metallization. Finally, another step of angle evaporation defines the second flare. This probe is illuminated from the top with a free-space laser beam and creates a near-field spot at its apex.

In order to assess the viability of such a probe for near-field microscopy, including the effects of its 3-D geometry, we have built a scale model of a $\mathrm{SiO}_{x} \mathrm{~N}_{y}$ cantilever and tip used with $10.6-\mu \mathrm{m}$ laser radiation. This scale model was built with the correct angles of $\mathrm{KOH}$ silicon etching in mind and can accurately model an actual probe with the metal patches being in the correct planes, at a $70^{\circ}$ angle to each other. The refractive index $n$ of the scale model material at the scaled frequency must be the same as that of the silicon-oxy-nitride at the frequency corresponding to a wavelength of $10.6 \mu \mathrm{m}$. The refractive index of $\mathrm{SiO}_{x} \mathrm{~N}_{y}$ depends upon fractions $x$ and $y$, and Modreanu $e t$ al. [35] show it to vary between 1.5 to 2.2 depending on these values. We measure $n=1.9 \pm 10 \%$ up to $18 \mathrm{GHz}$ for the acrylic material in our model, making it ideal for modeling silicon-oxy-nitride. We choose a scale factor of $10^{4}$, which gives a scaled frequency of $3 \mathrm{GHz}$ and a corresponding free-space wavelength of $\lambda=10 \mathrm{~cm}$. The scale model is shown in Fig. 3 with a schematic of the measurement setup as inset. The base length of the pyramid is $5 \mathrm{~cm}$ and the thickness of the acrylic is $5 \mathrm{~mm}$. This scales to a pyramid side length of $5 \mu \mathrm{m}$ and a membrane thickness of $0.5 \mu \mathrm{m}$, which are reasonable values for microfabrication.

To test the model, a dipole probe is coupled to a power meter and scanned over the pyramid tip as shown in the inset of Fig. 3 . A pyramidal horn is used with a $C$-band waveguide at $3.25 \mathrm{GHz}$ to launch a plane wave in the $x$-direction at the model. This frequency is used to accommodate the $C$-band waveguide and 

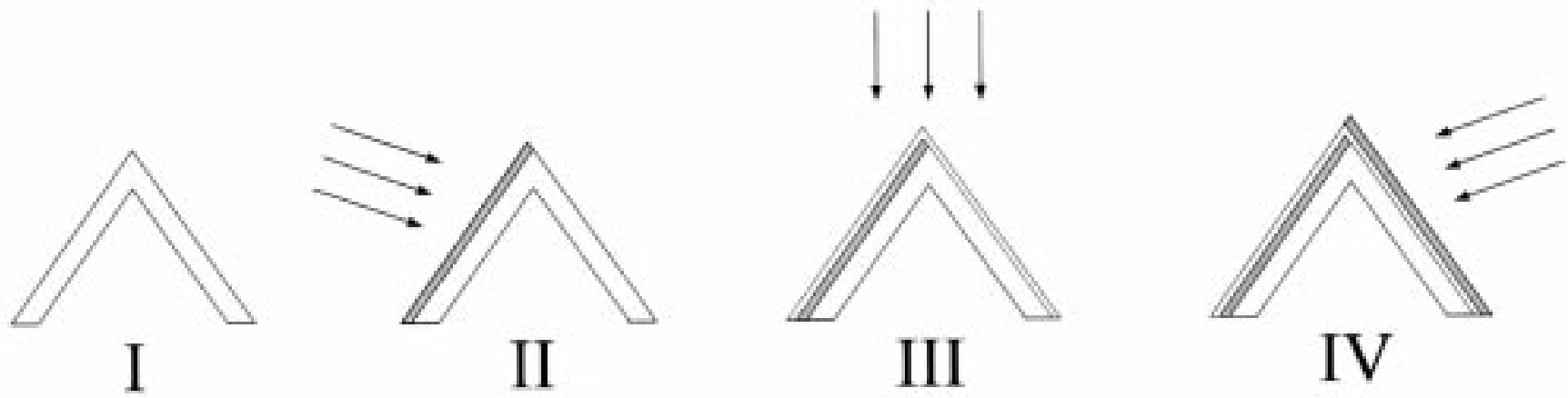

Fig. 2. Maskless fabrication technique for bowtie antennas with nanometer gaps on a SPM cantilever and tip. (I): Starting with a standard silicon-oxy-nitride tip (white), metal is shadow-evaporated (II) from one side first (gray). (III): A thin layer of tip material is deposited on the structure, defining the gap between antenna flares. (IV): Shadow evaporation from the other side forms the second metal patch.

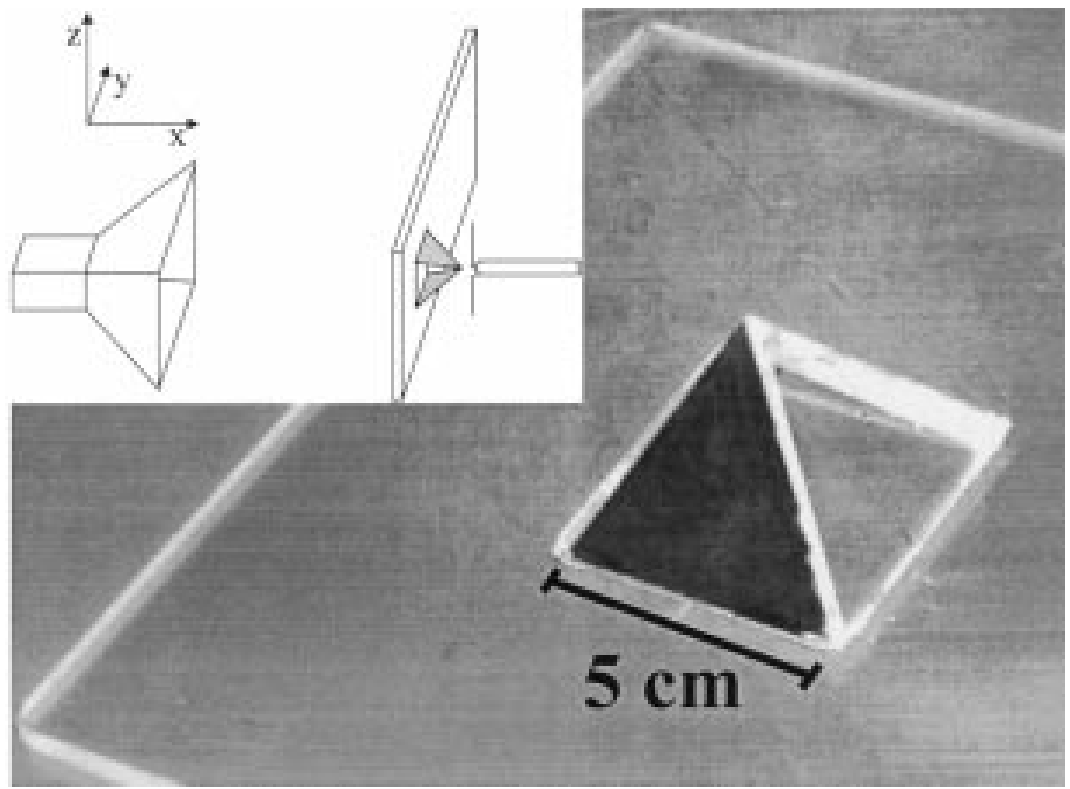

Fig. 3. Photograph of the scale model with part of the cantilever and tip shown. The dark area is one half of the bowtie antenna, which is defined with copper tape. The second flare of the antenna is on the other side of the pyramid (not visible). Inset: Schematic of the experimental setup, which simulates an FIR laser or other far-field source illuminating the scanning antennas: shown are horn, cantilever, and dipole antenna of the detector.

is not significantly higher than the scaled frequency. The distance between the horn and the scale model is $3 \mathrm{~m}$ to ensure far-field illumination. The dipole probe is $3 \mathrm{~mm}(\sim \lambda / 30)$ away from the apex of the pyramid. The dipole probe and the electric field of the wave emanating from the horn are oriented along the $z$-direction. The scale model is positioned such that the apex of the pyramid is at the zero position in the scan $(y)$ direction. In Fig. 4(a), multiple traces are plotted showing power detected by the dipole probe as it is scanned with and without metallization to prove the field enhancement expected with an antenna structure. Asymmetry in detected power about the zero position is evident. For the case with probe metallization, two sides of the pyramid were covered with copper tape. Fig. 4(b) shows the result of normalizing the power data measured with metallization by data with no copper tape. From this figure, it is apparent that the observed asymmetry is due to the styrofoam mount and the dielectric cantilever model. Clearly, a near-field spot with subwavelength extension is apparent in Fig. 4(b). Orienting the incident electric field perpendicular to this direction does not re- sult in field enhancement, hence we observe polarized antenna action in Fig. 4. Although the antenna tines were $2.25 \mathrm{~cm}$ long each, a sizable fraction of the wavelength, along the scan direction the antenna dimension is very small, resulting in deep subwavelength resolution. Lithographically defined antennas have already been used for infrared far-field applications [36], [37]. Together with the results presented here, this offers a clear path toward using near-field antennas in the IR and FIR.

\section{Using Planar Antennas In the Visible}

In principle, the use of antennas should be extendable to the visible and ultraviolet region, but there are physical limitations to scale modeling that prevent straightforward application of antennas beyond the difficulty of building them at the appropriate size. One limitation is that in scale models, metals are assumed to be almost perfect conductors, but as the frequency approaches the plasma resonance, they become transparent [38]. This resonance frequency usually lies in the UV, while some metals 


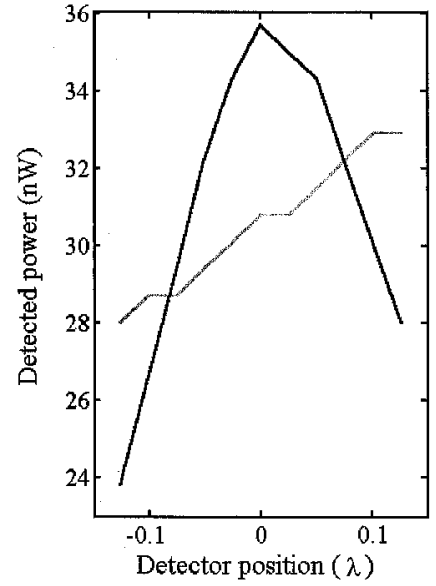

(a)

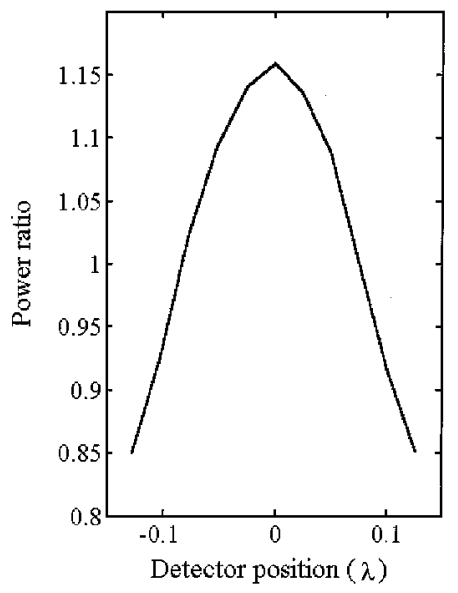

(b)

Fig. 4. Measured data points from the scale model of Fig. 3; the lines are guides to the eye. (a) Power detected by the dipole probe along the cantilever model with (black line) and without (gray line) the bowtie antenna metallization. (b) Ratio of the two data sets in (a), demonstrating the field-enhancement effect of the tip metallization pattern.

like Au have their resonance in the visible. Clearly, scale modeling will fail when approaching these frequencies, though in the infrared, Rutledge et al. [39] report similar patterns for cat whisker antennas using an RF scale model and an actual device. A second limitation is that the surface impedance is higher for higher frequencies because of the skin effect [36], [39]. The reactance of the surface impedance dominates the resistive part at higher frequencies, which complicates antenna behavior. This leads to difficulties in numerical modeling of antennas and deviations from experimental scale modeling.

Apart from these physical limitations, there are practical problems when fabricating antennas for near-infrared and visible frequencies. One problem that decreases efficiency is that of losses and slab modes associated with the finite thickness of the substrate. While material quality affects dielectric losses, mode losses can be minimized by using a substrate that is thinner than the optimal thickness [40] $h_{\text {opt }}$ given by

$$
h_{\mathrm{opt}} \leq \frac{\lambda_{0}}{4 \sqrt{\varepsilon_{r}-1}}
$$

where $\lambda_{0}$ is the vacuum wavelength and $\varepsilon_{r}$ is the relative dielectric constant of the substrate. For infrared and longer wave-

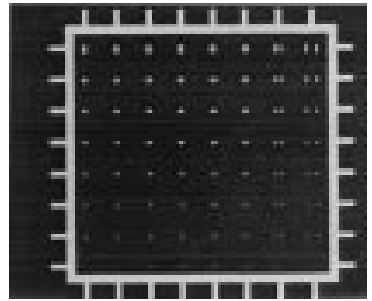

(a)

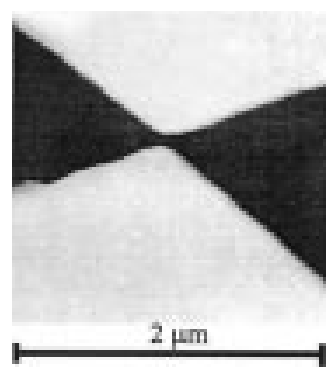

(b)

Fig. 5. (a) An antenna mask defined by electron beam lithography. (b) Scanning force microscope image of one antenna; the light areas correspond to metal, and the gap is $<70 \mathrm{~nm}$.

lengths, this is possible for cantilever-mounted antennas. For example, using $\lambda=10.6 \mu \mathrm{m}$ and $\mathrm{SiO}_{x} \mathrm{~N}_{y}$ as substrate $\left(\varepsilon_{r}=4\right)$, the optimal thickness would be $1.53 \mu \mathrm{m}$ which is close to the actual thickness of free-standing $\mathrm{SiO}_{x} \mathrm{~N}_{y}$ membranes used for SFM probes. For optical wavelengths, however, $h_{\mathrm{opt}}$ would be less than $100 \mathrm{~nm}$, which does not yield structures with sufficient mechanical stability. A second possibility to suppress such losses is to sandwich the antenna between two layers of substrate [41], the top cover being much thicker than $h_{\text {opt }}$. This would obviously interfere with the application of this antenna as near-field probe since the near-field region would be removed from the DUT. Since such a sandwich structure is not possible, the ratio of radiation into the dielectric versus air scales generally as $\varepsilon_{r}^{3 / 2}$ [36], so it is important to use a substrate with a very-low index of refraction for optical antennas. A third issue is that of surface roughness, which quickly becomes comparable to $\lambda$ at infrared and optical frequencies. A wide range of values is reported for the complex dielectric constant of the same metal, depending on purity and fabrication process of the metal film, making accurate characterization and modeling of these metal films difficult. Finally, when evaporating small, thin metal islands, oxidation can drastically limit their effectiveness as antennas. While $\mathrm{Au}$ is oxidation resistant, it does not have ideal optical properties to be used in the visible. $\mathrm{Al}$ and $\mathrm{Ag}$, on the other hand, have good optical properties but are known for their high rate of oxidation.

To take steps toward building antennas for the visible, we pattern a quartz mask with lithographically defined optical antennas and probe it directly (rather than using it to pattern another substrate, losing detail) using both a commercial fiberbased NSOM [42] and an experimental scattering-type NSOM setup, both in transmission and reflection. The mask and some of its features are shown in Fig. 5(a), and a closeup of one bowtie antenna is shown in Fig. 5(b). For high definition, electron beam lithography and metal lift off are used to create arrays 
of 75-nm-thick $\mathrm{Al}$ antenna patterns. Although the antennas have a wide range of flare angles and gaps, maximizing the chances for discovering an effective combination, we observe only slight field concentration effects and interference patterns parallel to the metal edges indicative of standing waves. In addition to the numerous loss mechanisms cataloged above, the conducting tip used for the scattering NSOM in this experiment might influence the radiation properties of the planar antennas as well.

For future work, the lithography process has to define sharper structures with smaller gaps than those in Fig. 5. Another important change will be to reduce the thickness of the metal layer. A thick layer has two disadvantages: since the near-field region extends in $z$ direction roughly as far as it does in the $x$-y plane, the sample (or DUT) is effectively removed from part of the near field if the gap is smaller than the metal is thick. The same is true if the antenna is not used to scan a sample but is scanned itself by an NSOM. In this case, the NSOM cannot see a large part of the near field because its probe cannot penetrate deeply enough into the gap. Furthermore, evaporating a thick layer gives the antenna a more rounded shape in its vertical profile, reducing field concentration close to the tip-sample interface.

\section{SUMMARY}

We examine the concept of using antennas integrated with SPM cantilevers for near-field microscopy, and in particular discuss scale modeling of a near-field probe using a silicon-oxy-nitride cantilever and tip with bowtie-patterned metallization on it to be used as a far-field to near-field transducer, demonstrating subwavelength field enhancement at the tip. Finally we discussed limits to scale modeling and fabrication for applications of antennas in the visible. We conclude that state-of-the-art control over metal dimensions and surface quality will be necessary to achieve antenna effects in the visible.

\section{ACKNOWLEDGMENT}

The authors wish to thank F. Keilmann, R. Hillenbrand, and B. Knoll at the Max-Planck-Institute for Biochemistry in Martinsried, Germany for performing scattering NSOM measurements and for valuable discussions. The authors are indebted to A. la Rosa, now at Portland State University, Portland, OR, for initial NSOM measurements. They thank M. Cywiak and C. J. Martínez at the Centro de Investigaciones en Optica A.C., Mexico, for cutting quartz samples for the optical antenna experiment.

\section{REFERENCES}

[1] F. Keilmann, "FIR Microscopy," Infrared Phy. Technol., vol. 36, no. 1, pp. 217-224, 1995.

[2] B. Knoll and F. Keilmann, "Near-field probing of vibrational absorption for chemical microscopy," Nature, vol. 399, pp. 134-137, 1999.

[3] — , "Infrared conductivitiy mapping for nanoelectronics," Appl. Phys. Lett., vol. 77, no. 24, pp. 3980-3982, 2000.

[4] A. Hammiche, H. M. Pollock, M. Reading, M. Claybourn, P. Turner, and K. Jewkes, "Photothermal FTIR spectroscopy: A step towards FT-IR microscopy at a resolution better than the diffraction limit," Appl. Spectrosc., vol. 53, no. 7, pp. 810-815, 1999.

[5] E. A. Synge, "A suggested method for extending microscopic resolution into the ultra-microscopic region," Philos. Mag., vol. 6, pp. 356-362, 1928.
[6] E. A. Ash and G. Nicholls, "Super-resolution aperture scanning microscope," Nature, vol. 237, no. 5357, pp. 510-512, 1972.

[7] E. Tanabe and W. T. Joines, "A nondestructive method for measuring the complex permittivity of dielectric materials at microwave frequencies using an open transmission line resonator," IEEE Trans. Instrum. Meas., vol. 25 , p. 222,1976

[8] D. Bérubé, F. M. Ghannouchi, and P. Savard, "A comparitive study of four open-ended coaxial probe models for permittivity measurements of lossy dielectric/biological materials at microwave frequencies," IEEE Trans. Microwave Theory Tech., vol. 44, pp. 1928-1934, Oct. 1996.

[9] D. E. Steinhauer, C. P. Vlahacos, S. K. Dutta, B. J. Feenstra, F. C. Wellstood, and S. M. Anlage, "Quantitative imaging of sheet resistance with a scanning near-field microwave microscope," Appl. Phys. Lett., vol. 72, p. 861, 1998.

[10] M. Abu-Teir, M. Golosovsky, D. Davidov, A. Frenkel, and H. Goldberger, "Near-field scanning microwave probe based on a dielectric resonator," Rev. Sci. Instrum., vol. 72, no. 4, pp. 2073-2079, 2001.

[11] G. Binnig, C. F. Quate, and C. Gerber, "Atomic force microscope," Phys. Rev. Lett., vol. 56, no. 9, pp. 930-933.

[12] G. Binnig and H. Rohrer, "Scanning tunneling microscopy," Helv. Phys. Acta, vol. 55, no. 6, pp. 726-735, 1982.

[13] D. W. van der Weide, "Localized picosecond resolution with a near-field microwave/scanning-force microscope," Appl. Phys. Lett., vol. 70, no. 6, pp. 677-679, 1997.

[14] D. Pohl, W. Denk, and M. Lanz, "Optical stethoscopy: Image recording with resolution $\lambda / 20$," Appl. Phys. Lett., vol. 44, no. 7, pp. 651-653, 1984.

[15] U. Dürig, D. Pohl, and F. Rohner, "Near-field optical-scanning microscopy," J. Appl. Phys., vol. 59, no. 10, pp. 3318-3327, 1986.

[16] A. Harootunian, E. Betzig, M. Isaacson, and A. Lewis, "Super-resolution fluorescence near-field scanning optical microscopy," Appl. Phys. Lett., vol. 49, no. 11, pp. 674-676, 1986.

[17] E. Betzig, A. Lewis, A. Harootunian, M. Isaacson, and E. Kratschmer, "Near-field scanning optical microscopy (NSOM)," Biophys. J., vol. 49, p. $269,1986$.

[18] E. Betzig and J. K. Trautman, "Near-field optics: microscopy, spectroscopy, and surface modification beyond the diffraction limit," Science, vol. 257, pp. 189-195, 1992.

[19] D. Courjon and C. Bainier, "Near field microscopy and near field optics," Rep. Progr. Phys., vol. 57, p. 989, 1994.

[20] F. Zenhausern, M. P. O'Boyle, and H. K. Wickramasinghe, "Apertureless near-field optical microscope," Appl. Phys. Lett., vol. 65, no. 13, pp. $1623-1625,1994$.

[21] R. Hillenbrand and F. Keilmann, "Complex optical constants on a subwavelength scale," Phys. Rev. Lett., vol. 85, no. 14, pp. 3029-3032, 2000 .

[22] M. Labardi, S. Patane, and M. Allegrini, "Artifact-free near-field optical imaging by apertureless microscopy," Appl. Phys. Lett., vol. 77, no. 5, pp. 621-623, 2000

[23] S. S. Osofsky and S. E. Schwarz, "Design and performance of a non-contacting probe for measurement on high-frequency planar circuits," IEEE Trans. Microwave Theory Tech., vol. 40, pp. 1701-1708, Aug. 1992.

[24] M. Golosovsky and D. Davidov, "Novel millimeter-wave near-field resistivity microscope," Appl. Phys. Lett., vol. 68, no. 11, pp. 1579-1581, 1996.

[25] A. Lahrech, R. Bachelot, P. Gleyzes, and A. C. Boccara, "Infrared-reflection-mode near-field microscopy using an apertureless probe with a resolution of 600," Opt. Lett., vol. 21, no. 17, pp. 1315-1317, 1996.

[26] B. Knoll and F. Keilmann, "Scanning microscopy by mid-infrared nearfield scattering," Appl. Phys. A, vol. 66, pp. 477-481, 1998.

[27] T. Nakano and S. Kawata, "Infrared evanescent-field microscope using CO2 laser for reflectance measurement," Optik, vol. 94, no. 4, pp. $159-162,1993$.

[28] D. W. van der Weide, U.S. Patent 5936 237, 1999.

[29] M. Fee, S. Chu, and T. W. Hänsch, "Scanning electromagnetic transmission line microscope with sub-wavelength resolution," Opt. Commun., vol. 69, no. 3, 4, pp. 219-224, 1989.

[30] F. Keilmann, U.S. Patent 4994 818, 1991.

[31] D. W. van der Weide and P. Neuzil, "The nanoscilloscope: combined topography and AC field probing with a micromachined tip," J. Vac. Sci. Technol. B, vol. 14, no. 6, pp. 4144-4147, 1996.

[32] A. Kramer, F. Keilmann, B. Knoll, and R. Guckenberger, "The coaxial tip as a nano-antenna for scanning near-field microwave transmission microscopy," MICOB, vol. 27, p. 413, 1996.

[33] R. D. Grober, R. J. Schoelkopf, and D. E. Prober, "Optical antenna: towards a unity efficiency near-field optical prode," Appl. Phys. Lett., vol. 70, no. 11, pp. 1354-1356, 1997. 
[34] R. D. Grober, U.S. Patent 5696372.

[35] M. Modreanu, N. Tomozeiu, P. Cosmin, and M. Gartner, "Physical-optical properties of LPCVD amorphous silicon rich-nitride and oxynitride," presented at the IEEE Semiconductor Conference CAS'98 Proceedings, 1998.

[36] E. N. Grossman, "Lithographic antennas for submillimeter and infrared frequencies," presented at the IEEE International Symposium on Electromagnetic Compatibility, 1995.

[37] C. Fumeaux, W. Herrmann, F. K. Kneubühl, H. Rothuizen, B. Lipphardt, and C. O. Weiss, "Nanometer thin-film Ni-NiO-Ni diodes for mixing 28 $\mathrm{THz} \mathrm{CO}_{2}$-laser emissions with difference frequencies up to $176 \mathrm{GHz}$," Appl. Phys. B, vol. 66, pp. 327-332, 1998.

[38] J. D. Jackson, Classical Electrodynamics, 3rd ed. New York, : Wiley, 1999.

[39] D. B. Rutledge, S. E. Schwarz, and A. T. Adams, "Infrared and submillimeter antennas," Infrared Phys., vol. 18, pp. 713-729, 1978.

[40] D. Mirshekar-Syahkal and D. Wake, "Bow-tie antennas on high dielectric substrates for MMIC and OEIC applications at millimetre-wave frequencies," Electron. Lett., vol. 31, no. 24, pp. 2060-2061, 1995.

[41] A. Moussessian and D. B. Rutledge, "A millimeter-wave slot-V antenna," in Proc. IEEE Antennas Propagat. Soc. Int. Symp., vol. 4, 1992, pp. 1894-1897.

[42] TM Microscopes [Online]. Available: http://www.tmmicro.com

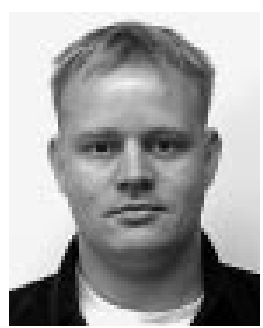

Björn Rosner (S'02) received the M.S. degree in physics from the University of Delaware, Newark, in 1998. He is currently working toward the Ph.D. degree at the same university.

His research interests include NEMS sensor fabrication, application of microwave near-field probes and the interactions of light with metals and dielectrics.

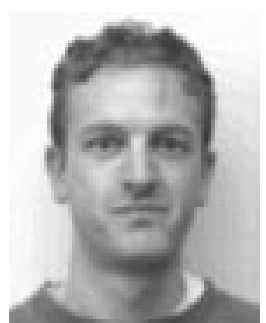

John Peck (S'01) received the B.S. and M.S. degrees in physics from the University of Wisconsin-Madison. He is currently working toward the $\mathrm{Ph} . \mathrm{D}$. at the same university.

His research interests include microwave dielectric spectroscopy, planar antennas, and near-field probes.

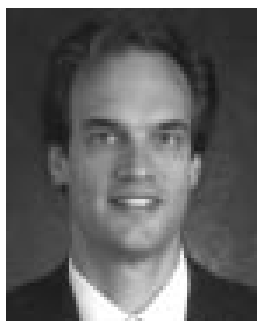

Daniel van der Weide (S'86-M'87) received the $\mathrm{Ph} . D$. degree in electrical engineering form Stanford University, Palo Alto, CA, in 1993.

$\mathrm{He}$ is currently an Associate Professor in the Department of Electrical and Computer Engineering, University of Wisconsin-Madison, where he works in micromachined high-frequency electromagnetic sensors; localized microwave spectroscopy on biomolecules and semiconductor devices using multifunctional scanning probes and antennas; and terahertz spectroscopy with integrated antennas and nonlinear trasmissionlines. He cofounded and directed the Center for Nanomachined Surfaces (CNS) at the University of Delaware, Newark, where he was and Assistant and the Associate Professor from 1995 to 1999.

Dr. Van der Weide is the recipient of the NSF Presidential Early Career and ONR Young Investigator Award. 